\title{
An Analysis of the Debate over Structural Universals
}

\author{
Pawel GARBACZ \\ Department of Philosophy, John Paul II Catholic University of Lublin, Poland
}

\begin{abstract}
The paper outlines a conceptual framework to identify all ontological and logical aspects relevant for the debate over structural universals. The framework allows for a multi-facetted classifications of various accounts of the latter and facilitates their comparison in a systematic way. To show the framework in action I use it to classify all major theoretical positions in this debate.
\end{abstract}

Keywords. structural universal, mereology, instantiation, metaontology

\section{Introduction}

The concept of universals is a key explanatory device in philosophy and in applied ontology. The distinction between universals and individuals is one of the most fundamental distinctions one can make in any discourse or over any domain. Evoking universals one may want to explain why two individual entities are similar, why we observe certain regularities among different, although similar individuals, etc. Universals can also play more specific theoretical roles, e.g., they may be possible worlds in ersatz modal realism. Thus universals come in handy, but at a price since they seem to be different in many respects from the individual entities and these differences may give rise to various paradoxes or antinomies. One of the less known objections questions their theoretical value on the basis of the following argument, which was probably first explicitly stated in [1]:

1. If there are universals, then, given what we know about the universe, it is possible that all universals are structural, i.e., that all universals have parts 1 or some kind of structure built out of other universals.

2. The concept of structural universals is not intelligible.

[1] focusses mainly on the second premise arguing that one cannot provide a consistent and meaningful account of the mereological structure of universals. So, prima facie, it casts a shadow of doubt on structural universals. Still, the argument can be seen as a conditional refutation of all kinds of universals, where the condition in question describes a possibility of the infinite complexity, i.e., the possibility that every universal is composed of other universals. Given this provision, the argument builds a case against the concept of universals questioning the intelligibility of just one kind of universals, namely, structural universals. Structural universals are claimed to be faulty devices because one

\footnotetext{
${ }^{1}$ I assume that 'part' means always 'proper part' throughout this paper.
} 
cannot rationally account for their mereological structure, i.e., one cannot explain what it means that one universal is part of another. And this is all what structural universals are about. Therefore, if the universe turns out to be infinitely complex and all universals are structural, then no universal is intelligible and as such cannot serve any explanatory role. As a matter of fact, this particular type of argument, if conclusive, effectively undermines all views that posit any type of universals other than nominalistic (i.e. linguistic) constructs. In other words, both realism and conceptualism about universals, of any kind, must go.

Needless to say such sweeping objection gave rise to a number of accounts that attempted to salvage universals as bona fide theoretical devices. These attempts usually aim to rebut Lewis's criticism by explaining what it means that one universal is part of another and by explaining away the issues he raised in [1].

A careful analysis of the ensuing discussion reveals that the problem is, so to speak, multi-facetted, i.e., that it involves a number of more fundamental issues. In this paper I attempt to identify these aspects by means of a conceptual framework in which all these different accounts of structural universals can be accommodated so that one can classify and compare them. In particular my goal is to mine the elementary points of contention therebetween so that it will be more transparent what it is at stake in this debate.

Let me start the analysis with fleshing out the reasons because of which [1] questions the intelligibility of structural universals. ${ }^{2}$

\section{Problems}

A structural universal is a universal that is complex, i.e., it is somehow composed of other universals ${ }^{3}$ The controversy over structural universals concerns this very qualification 'somehow': what does it actually mean that a universal is composed out of other universals.

As usual, it is easier to say what it does not mean. [2] states upfront that structural universals are not conjunctive universals, where the latter are defined as below:

Definition 1 (Conjunctive Universals) Universal $\mathrm{U}$ is conjunctive iff given that $\mathrm{U}$ is composed of universal $\mathrm{V}(\mathrm{U} \neq \mathrm{V})$, then for every individual $x$, if $x$ instantiates $\mathrm{U}$, then $x$ instantiates $\mathrm{V}$ as well.[2]

So, for example, universal RED AND ROUND is conjunctive provided that we assume that it is composed of universal RED and universal ROUND 4

Given that structural universals don't boil down to conjunctive universals, what are they then? Consider an ethane $\left(\mathrm{C}_{2} \mathrm{H}_{6}\right)$ molecule, i.e., an individual that is composed of six hydrogen (individual) atoms and two (individual) carbon atoms as depicted in fig. 1 If we grant that universals exist, this molecule instantiates one of them: ETHANE. But then also all its hydrogen atoms and the two carbon atoms instantiate some universals,

\footnotetext{
${ }^{2}$ I would like to thank the anonymous reviewers of this paper whose comments allowed me to improve its quality.

${ }^{3}$ For the sake of necessary simplifications this paper ignores the temporal aspect of parthood or any akin relationship, both in the domain of universals and in the domain of individuals.

${ }^{4}$ As usual, some would disagree: the theory outlined in [3] describes all structural universals as conjunctive universals.
} 
<smiles>CC</smiles>

Figure 1. Ethane

scilicet, HYDROGEN and CARBON, respectively. Now each (individual) hydrogen atom is part of the ethane molecule. Is HYDROGEN part of METHANE as well? Obviously, there is some relation, at least akin to parthood, which relates these two, but if it is a species of parthood, then it is a rather peculiar one.

First, different structural universals may have the same universals as parts. For example, ETHANE has two conformational isomers - depicted in fig. 2

1. STAGGERED ETHANE

2. ECLIPSED ETHANE

which have the same universals as parts and which are different due to the different relative positions of those parts. Thus, this type of parthood is not extensional 5

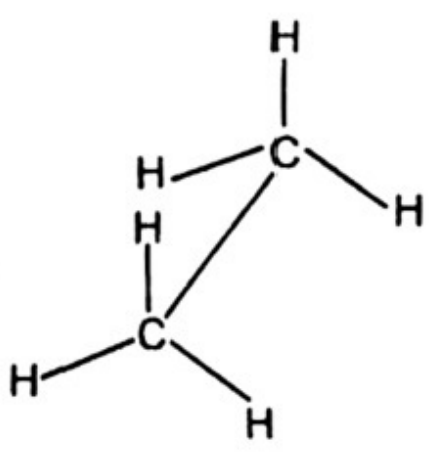

Eclipsed ethane|

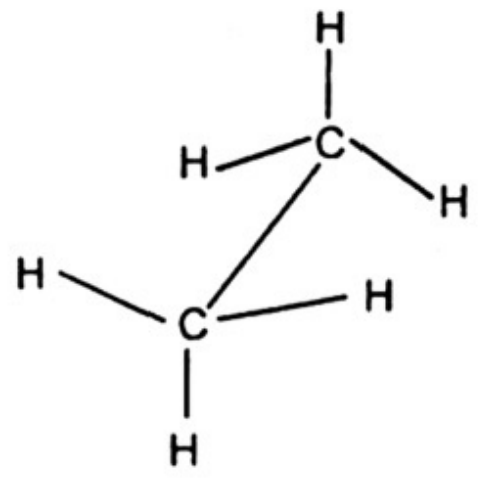

Staggered ethane

Figure 2. Ethane conformations

Secondly, compare ETHANE to METHANE $\left(\mathrm{CH}_{4}\right)$.<smiles>C</smiles>

Figure 3. Methane

\footnotetext{
${ }^{5}$ Relation $R$ is extensional if and only if it is the case that $\forall x, y, z\{[R(z, x) \equiv R(z, y)] \rightarrow x=y\}$.
} 
One may wish to say METHANE, in the same way as ETHANE, has HYDROGEN and CARBON universals as parts, i.e., that HYDROGEN and CARBON are parts of both ETHANE and METHANE. At the same time ETHANE is different from METHANE, but, it seems to me, in a different way than STAGGERED ETHANE is different from ECLIPSED ETHANE. They are different not because their parts are arranged in different ways but because they have different parts. One may say that ETHANE is different from METHANE because (i) CARBON occurs twice in the former and only once in the latter and (ii) HYDROGEN occurs six times in the former and four times in the latter. Or one may say that the difference between ETHANE to METHANE is due to the fact that (i) the former has two CARBON parts and the latter has just one and that (ii) the former has six HYDROGEN parts and the latter has four of them. In other words, CARBON is part of ETHANE twice over and is part of METHANE only once (and similarly for HYDROGEN). Now this problem may be seen as more substantial than the first one: what can it mean for something to have a part twice over and how is this different from having it only once or from having it, say, three times over?

Thirdly, note that if structural universals do have parts, then we may need to recognise a number of unexpected mereological consequences. For example, if HYDROGEN is part both of METHANE and ETHANE, then the universals of METHANE and ETHANE overlap although none of their instances do.

\section{Solutions}

These issues, well at least the first two, were originally stated in [1], where D. Lewis also outlines a number of possible solutions. His outlines are rather sketchy and as such require a certain amount of rational reconstruction in the sense of $\mathrm{R}$. Carnap.

Let me start with the so-called pictorial theory of structural universals. The main tenet of this theory is that individuals are isomorphic (with respect to the relation of parthood) to the universals they instantiate in the sense due to which representations like fig. 1 or fig. 3 construed as "pictures", illustrate the mereological structure of both the universal and its instances. As a matter of fact, the details of the pictorial theory of structural universals may require a more convoluted description:

Solution 1 (Pictorial Theory) If individual $x$ instantiates structural universal $\mathrm{U}$, then there is a set $X$ of $x$ 's (proper) parts such that

1. $x$ is the mereological sum of $X$;

2. there exists a surjective map $f$ from $X$ to the multiset of $U$ 's parts such that

(a) for all $y, z \in X$ if $y$ is part of $z$, then $f(y)$ is part of $f(z)$;

(b) $f$ corresponds to the relation of instantiation, i.e., for all $x \in X, f(x)$ is the universal $x$ instantiates 6

Clause 1, or rather the whole idea of taking some, i.e. not necessarily all, parts of individuals into account when we consider the morphism between a universal and its instances, is justified by the need of being able to represent a broad range of universals and

\footnotetext{
${ }^{6}$ For the sake of simplicity, I assume here that the relation of instantiation is a function, i.e., that no individual can instantiate more than one universal.
} 
all kinds of morphisms. Consider the universal of METHANE. Prima facie it contains universals of HYDROGEN and CARBON atoms but it does not contain universals corresponding to their subatomic parts, e.g., to electrons or protons. If this, somewhat superficial, interpretation is right, then the universal of METHANE specifies, so to speak, its parts just down to the level of atoms and the structural similarity between an individual molecule of methane and METHANE does not range over all parts of the former, i.e., an instance of a universal may have parts that are not mapped onto any part of the universal. The clause in question is to allow for such cases - as it allows also for all cases where all parts of an individual are mapped onto its universal's parts. In other words, this clause is to make room for structures being homo- or isomorphic down to a certain level of granularity.

Now Pictorial Theory may occur in two versions:

1. weak: where there exists no bijective map that satisfies clause 2 in solution 1 .

2. strong: where there exists a bijective map that satisfies clause 2 in solution 1

Given a weak version one may infer, along the lines outlined in the previous section, all three issues mentioned there:

1. parthood among universals is not extensional;

2. one universal can be part of another multiple times;

3. many structural universals overlap.

That is to say, one may infer these consequences within the context of the weak version the pictorial theory, i.e., the arguments present previously are consistent with this version.

I reckon that the theory developed in [4] could be classified as an example of such account although the main body of this approach is focused on how to represent mereological structure of individuals.

Given the strong version of the pictorial theory corollary 2 drops out because in this version there are as many parts of a structural universal as there are parts of its instances and no such part need to be part of the universal more than once. An example of such approach is described in [5], where each universal that is part of a structural universal is claimed to be a universal that is a subtype of one common or global universal. The idea is that each occurrence of a universal in a structural universal is actually a universal, which is a kind of local species of one common genus. This account would, for instance, have it that (i) there exists the HYDROGEN universal, (ii) the METHANE structural universal would have as its parts six universals that are subtypes of HYDROGEN, and (iii) each individual hydrogen atom in a methane molecule is an instance of one of these six universals. In this particular exemplification of the strong pictorial theory even corollary 3 no longer holds because [5] has it that each structural universal has its "own", local subtypes, so ETHANE and METHANE have no part in common. The non-extensionality of parthood still remains an issue here given the existence of conformational isomers. But the proponent of the strong theory will have to address the more serious objection, mainly the problem of egregious proliferation of universals.

Another type of solutions questions the claim that structural universals have parts. Now it is argued that a non-mereological account of structural universals is needed a version of such solution was discussed in [1] as the magical theory. Such solutions take it for granted that if the notion of structural universal is to be different from the more general notion of universal simpliciter, there should exist some differentia specifica, 
which would separate structural from non-structural universals. This difference needs to be rendered in terms of a certain relation between structural universals and universals so that a universal is structural if there are one or more universals that bear this relation to it. For example, [1] tentatively uses the term "involvement", i.e., a universal is structural if it involves other universals. If we subscribe to a non-mereological account of this type, we need to be able to identify a certain relation by means of which structural universals may be defined. Let $\mathrm{R}$ be the appropriate relation. This type of solution, which incidentally generalises the pictorial theory, would require that we adapt clause 2 in solution 11 as follows:

Solution 2 (Magical Theory) If individual $x$ instantiates structural universal $\mathrm{U}$, then there is a set $X$ of $x$ 's (proper) parts such that

1. $x$ is the mereological sum of $X$;

2. there exists a surjective map $f$ from $X$ to the multiset of U's $\mathrm{R}$-chunks such that

(a) for all $y, z \in X$ if $y$ is part of $z$, then $\mathrm{R}(f(y), f(z))$;

(b) $f$ corresponds to the relation of instantiation, i.e., for all $x \in X, f(x)$ is the universal $x$ instantiates.7

As before this account may come in two versions: weak and strong. Given the weak version of the magical theory the three aforementioned observations still hold:

1. $\mathrm{R}$ is not extensional;

2. one universal can be R-chunk of another multiple times;

3. many structural universals R-overlap.

However this time given a proper account of $\mathrm{R}$ one can probably dismiss 1 and 3 as implicit features of R. Still 2 should be seen as an issue because no matter what kind of relation is at stake if you think about relations in the usual sense, it makes no sense to say that universal $U_{1}$ is $R$-ed to universal $U_{2}$ multiple times. But, as in the case of strong pictorial theory, 2 may drop out for the strong version of the magical theory if, for example, R-chunks of universals are construed as in [5].

Thirdly, one may raise a doubt whether parts of universals are themselves universals. Given the conceptual framework adopted in this section this amounts to dropping clause $2 \mathrm{~b}$ in solution 1 The resulting theory may be expressed in the homogeneous or heterogeneous sense: one may require that the mapping function never coincides with the relation of instantiation (see assumption 3 ) or require that it coincides with it only sometimes (assumption 4):

Solution 3 (Homogeneous Amphibian Theory) If individual $x$ instantiates structural universal $\mathrm{U}$, then there is a set $X$ of $x$ 's (proper) parts such that

1. $x$ is the mereological sum of $X$;

2. there exists a surjective map $f$ from $X$ to the multiset of $U$ 's parts such that

(a) for all $y, z \in X$ if $y$ is part of $z$, then $f(y)$ is part of $f(z)$;

(b) for all $x \in X, f(x)$ is not the universal $x$ instantiates.

\footnotetext{
${ }^{7}$ The notion of chunk is a generalisation of the notion of part: $x$ is an R-chunk of $y$ if $\mathrm{R}(x, y)$. Conversely, one may define R-lumps: $x$ is an R-lump of $y$ if $\mathrm{R}(y, x)$.
} 
Solution 4 (Heterogeneous Amphibian Theory) If individual $x$ instantiates structural universal $\mathrm{U}$, then there is a set $X$ of $x$ 's (proper) parts such that

1. $x$ is the mereological sum of $X$;

2. there exists a surjective map $f$ from $X$ to the multiset of $U$ 's parts such that

(a) for all $y, z \in X$ if $y$ is part of $z$, then $f(y)$ is part of $f(z)$;

(b) for some $x \in X, f(x)$ is not the universal $x$ instantiates and for some $x \in X$, $f(x)$ is the universal $x$ instantiates.

In both versions the amphibian theory has it that some parts of universals are not universals themselves. So on this approach four HYDROGEN entities in ETHANE universal are not universals themselves. Obviously, the main problem now is what these entities actually are. One may say that they are occurrences of universals, e.g., four items marked by $\mathrm{H}$ in fig. 3 are four different occurrences of the HYDROGEN universal. Again it is easier to say what these occurrences are not: i.e., occurrences of universals are neither individuals nor universals. [1]'s dismissively dubs them "amphibians" because he argues that they do not fit the standard ontological landscape where all entities are disjointly and exhaustively divided into individuals and universals. Also, given solutions like [5], one may ask how a "local universal" is different from an occurrence of the global universal. In any case I am not aware of any specific example of this kind of solution and that whether the three issues mentioned in the previous section apply now as well depends on its details.

Finally I should note that [1] mentions also the linguistic account of structural universals, where structural universals are (unspecified) set-theoretic constructions of words, or more specifically speaking, of predicates, which are simple, i.e., non-structural, universals. These constructions are said to follow the recursion patterns we know from the model theory, e.g., the relation of instantiation becomes now the relation of satisfaction. [1] does not provide us with the details of this account, e.g., we don't know whether words or predicates he mentions are word-tokens or word-types and we are not informed about the type(s) of the set-theoretical constructions in question, so we don't know whether simple universals can be construed as parts of the structural universals 8 As a result, it is not possible to unambiguously render this solution in terms similar to the other three solutions.

\section{Facets of Structural Universals}

All parties to the debate on structural universals seem to presuppose a number of relatively obvious assumptions:

1. There are two disjoint domains of entities: individuals and universals, where the former instantiate the latter.

\footnotetext{
${ }^{8}$ On the other hand, we do know why [1] does not accept this solution: it requires that all structural universals are ultimately grounded in simple universals and this excludes the possibility of the infinite complexity.
} 
2. Universals are posited, rather than discovered, to serve some theoretical goal(s), for example they can be deployed to explain various kinds of similarity between individuals, i.e., two individuals are similar to one another in a given respect if they both instantiate the universal relevant for this respect, e.g., they are similar as chemical molecules if they instantiate the same chemical formula.

3. The relation of parthood is primarily defined on individuals, i.e., there are certain mereological facts that can be asserted about them. These assertions can be deployed to explain some similarities among individuals, i.e., one may explain that two individuals are similar pointing out to the fact they have isomorphic or homomorphic mereological structures.

4. The relation of parthood or rather a counterpart thereof can be derivatively defined on universals, well at least on some of them. Namely, we saw in section 1 that there are universals that are posited to explain mereological similarities between their instances, i.e., the fact that one individual is mereologically similar to another individual is ontologically grounded in the universals these two individuals instantiate. Such similarities are not just accidental facts but that they hold between the individuals of necessity and that this kind of necessity is somehow grounded in the respective universals. The interplay between mereology and necessity can be described as follows: if individual $x$ is part of individual $y$ and if this mereological fact has to do with the universals $x$ and $y$ instantiate, say, respectively, $\mathrm{V}$ and $\mathrm{U}$, then necessarily, every instance of $\mathrm{V}$ is part of some instance of $\mathrm{U}^{9}$

5. If this account adequately represents the common assumptions of the debate in question, we are now in the position to provide a definition of structural universals, Here $\mathrm{U}$ is defined as a structural universal if and only if there exists (at least) one universal V such that the following two principles are satisfied by $\mathrm{U}$ and V:

Principle 1 It is possible that some instance of universal $\mathrm{U}$ has a part that is an instance of $\mathrm{V}, 10$

Principle 2 If some instance of universal $\mathrm{U}$ has a part that is an instance of universal $V$, then necessarily, every instance of $\mathrm{U}$ has a part that is an instance of $V 11$

If universals $\mathrm{U}$ and $\mathrm{V}$ satisfy these principles, one can say that $\mathrm{V}$ is part, or better chunk, of U.

Having the scope of agreement outlined let me elaborate the contestable aspects of the debate. The previous section showed that the issues in question go well beyond the initial Lewis' worries about the extensionality of parthood. In fact, it involves a system of more basic issues. Let me now untangle them by identifying and elaborating on the main aspects involved:

\footnotetext{
${ }^{9}$ This interpretation allows for mereological similarities between individuals that are not ontologically grounded in the universals these individuals instantiate.

${ }^{10}$ The modal qualifier is to allow for the case of uninstantiated structural universals.

${ }^{11}$ This principle is a slightly modified version of the CO-INT principle from [2].
} 
A. General structurality: this aspect concerns the issue whether the domain of universals can be seen as a structure, i.e., whether universals, including structural universals, are related to one another by some relation $\mathrm{R}$ that is relevant for explaining principle 2

- All accounts of structural universals I am aware of imply that structural universals are related to one another in some way, but generally speaking it is possible to hold an extreme view where principles of the form principle 2 are explained without a reference to any relationships between universals. One may hold a sort of brutalism (with respect to structural universals) according to which principle 2 reports just brute facts ${ }^{12}$ Alternatively, one may refer to the specific natures, i.e., intrinsic properties, of these universals. Be it as may, all other facets mentioned below are meaningful only if the general structurality question is answered in the positive.

- A structure in which a structural universal U occurs will be called a universal structure. Similarly, the mereological structure of an individual will be called individual structure.

B. Structure multiplicity: this concerns the issue whether a structural universal has a single structure or multiple structures.

- Most accounts of structural universals presuppose that a structural universal has the unique structure, be it mereological or otherwise, which is referred to when principle 2 is explained. However [7] points out to a theoretical possibility where a structural universal is described by means of two relations to other universals: (non-extensional) parthood and composition ${ }^{13}$ Also [8] mentions two kind of relations: "slothood" ('... is a slot in (universal) ...') and slot occupancy ('(universal) ... occupies (slot) ...').

- I assume that each structure in which a structural universal occurs is defined by one relation. Thus, multiple universal structures mean multiple relations $\mathrm{R} 1, \mathrm{R} 2, \ldots$ etc.

C. Structure type: this concerns the issue whether $\mathrm{R}$ is the relation of parthood or not.

- Following [1] I assume that the relation of parthood is extensional, so all nonextensional relations, even if conceptually close to parthood, will be classified here as other relations. I take this assumption as a terminological convention without any ontological or logical commitment.

D. Structural universal's role in structure: this concerns the issue whether structural universals play the role of R-lumps in these structures or play the role of R-chunks or neither.

- This aspect is to make room for all kinds of general non-mereological solutions in which structural universals play the role that generalises the mereological role of the part, or the role that generalises the role of the whole or neither.

\footnotetext{
${ }^{12}$ I have in mind here a theory similar, mutatis mutandis, to the theory of mereological composition exposed in [6].

${ }^{13}$ I assume here the interpretation of [7] given in [2].
} 
- I am not aware of any account where structural universals play the role of R-chunks, but [3] implies that structural universals play neither the role of R-chunks nor R-lumps.

E. Structural similarity: this concerns the issue whether universal structures are structurally similar to the individual structures of their instances or not.

- Structural similarity is given by a map $f$ from the set of instance's parts to the set of universal's $R$-chunks such that if individual $x$ is part of individual $y$, then $f(x)$ is $R$-chunk of $f(y)$. I take it that this map is a genuine ontological relation (or a natural relation in the sense of [9]), e.g., the relation of instantiation, and not just a set (of ordered couples).

- Again all known accounts of structural universals imply the affirmative answer to this question, i.e., they endorse the existence of some similarity map, but a view that universal structures are not similar to instance structures probably can be worked out. For example, if we interpret formal parts in the sense of [10] as such parts (of individuals) that either (i) are individuals that do not instantiate any universals or (ii) are individuals that instantiate universals that are not parts of any other universals, then the individual structures will not be similar to the respective universal structures. As before, all facets mentioned below are meaningful only if the structural similarity question is answered in the positive.

- If universal structures are structurally similar to the individual structures, then two other questions are relevant:

(a) Similarity ontological interpretation: this concerns the issue whether the similarity map coincides with the instantiation relation.

(b) Similarity formal interpretations:

i. Similarity scope: this concerns the issue whether the similarity map is a partial or (total) function - see the discussion of clause 1 of solution 1 above.

ii. Similarity formal properties:

A. one aspect is whether the similarity map is surjective or not;

B. another aspect is whether the similarity map is injective or not.

The aspects B. C. D. and E. are orthogonal to one another, i.e., an answer to any of these does not imply or exclude any answer to all others. Similarly, the aspects: E.a. E.b and the aspects: E.(b)iiA E.(b)iiB, are, respectively, independent from one another. The visual summary of all these facets is shown in fig. 4

\section{Solutions Classified}

Having the above conceptual framework in place we can now classify the major accounts of structural universals. I show in table 1 an attempt towards this end - obviously a rough and ready one because the proper classification would require a detailed conceptual analysis of each of these theories - which most likely would reveal alternative interpretations for at least some of them - and this task goes well beyond the scope of this paper. For the sake of illustration of possible intricacies let me note that [11]'s account seems to as- 


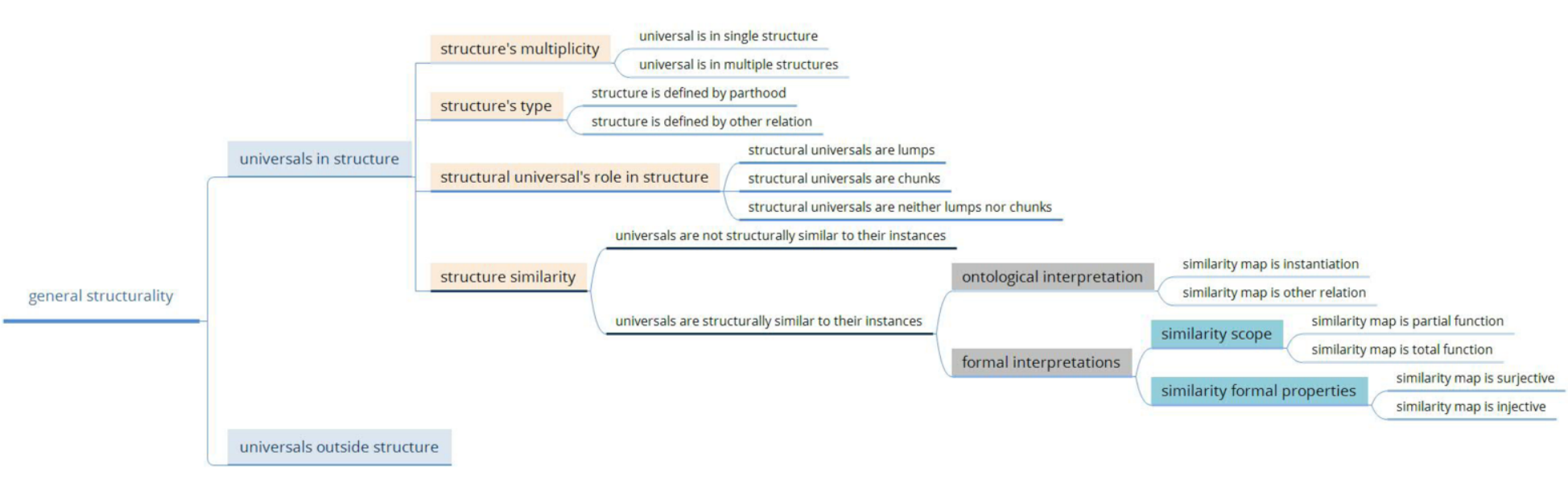

Figure 4. Main facets of the debate over structural universals 


\begin{tabular}{|c||c|c|c|c|c|}
\hline Source & Structure Multiplicity & Structure Type & Universal Role & $\begin{array}{c}\text { Similarity } \\
\text { Interpretation }\end{array}$ & $\begin{array}{c}\text { Similarity } \\
\text { Formal Properties }\end{array}$ \\
\hline \hline$[1]$ (Pictorial) & Single & Parthood & Lump & Instantiation & Surjection and Non-Injection \\
\hline$[4]$ & Single & Parthood & Lump & Instantiation & Surjection and Non-Injection \\
\hline$[5]$ & Single & Parthood & Lump & Instantiation & Surjection and Injection \\
\hline$[1]$ (Magical) & Single & Other Relation & Lump & Instantiation & Surjection and Non-Injection \\
\hline$[11]$ & Single & Non-extensional Parthood & Lump & Instantiation & Surjection and Injection \\
\hline$[3]$ & Single & Constitution & Neither & Instantiation & Surjection and Non-Injection \\
\hline$[7]$ & Multiple & $\begin{array}{c}\text { Non-extensional Parthood } \\
\text { Composition }\end{array}$ & Lump & Instantiation & Surjection and Non-Injection \\
\hline$[8]$ & Multiple & $\begin{array}{c}\text { slothood } \\
\text { Slot occupancy }\end{array}$ & Lump & Instantiation & Non-Surjection and Non-Injection \\
\hline$[1]$ (Amphibian) & Single & Parthood & Lump & Other Relation & Surjection and Non-Injection \\
\hline$[13]$ & Single & Parthood & Lump & Instantiation & Non-Surjection and Non-Injection \\
\hline$[14]$ & Single & Parthood & Lump & Instantiation & Non-Surjection and Non-Injection \\
\hline
\end{tabular}

Table 1. Classification of theories of structural universals 
sume a rather subtle distinction between universals that are state-of-affairs types ("... is $F ")$ and universals that are components of state-of-affairs types (" $F$ "), which distinction may involve two kinds of instantiation relationship or may hide the fact that the former universals are more like amphibians than like universals proper.

The classification does not include two of the aforementioned aspects:

1. general structurality: all theories in scope assume that structural universals have structures of some kind.

2. similarity scope: all theories in scope do not explicitly specify the scope, but implicitly they do assume partial scope for the examples they mention.

Let me note that the framework is capable to accommodate not just the existing solutions but it also allows for possible, yet-not-formulated, accounts of structural universals. On the other hand, it does not range over all solutions for the issues raised in [1], in particular all theories that "explain away" structural universals, like [12], are outside its scope.

\section{Conclusions}

The above analysis of the debate over structural universals showed a number of dependent and independent issues (of the ontological and formal provenance) that are relevant when one needs to formulate an adequate account of structural universals. These aspects go well beyond the initial problem of the extensionality of parthood raised by D. Lewis. All of them seem to be pertinent for the comprehensive evaluation of any theory of universals. Having them in place we can classify and compare different solutions in a more systematic way, although obviously not every detail of every account is captured in the framework. Finally the identified aspects may be part of a requirement specification for a logical framework that is capable to represent structural universals of any theoretical provenance.

\section{References}

[1] Lewis D. Against structural universals. Australasian Journal of Philosophy. 1986;64(1):25-46.

[2] Fisher ARJ. Structural universals. Philosophy Compass. 2018;13(10):e12518.

[3] Bigelow J, Pargetter R. A theory of structural universals. Australasian Journal of Philosophy. 1987;67(1):1-11.

[4] Masolo C, Vieu L. Graph-Based Approaches to Structural Universals and Complex States of Affairs. In: Formal Ontology in Information Systems - Proceedings of the 10th International Conference, FOIS 2018, Cape Town, South Africa, 19-21 September 2018; 2018. p. 69-82.

[5] Davis WA. On Occurrences of Types in Types. Australasian Journal of Philosophy. 2014;92(2):349-363.

[6] Marksonian N. Brutal Composition. Philosophical Studies. 1998;92:211-49.

[7] Hawley K. Mereology, Modality and Magic. Australasian Journal of Philosophy. 2010;88(1):117-133. 
[8] Bennett K. Having a Part Twice Over. Australasian Journal of Philosophy. 2013;91(1):83-103.

[9] Lewis D. New Work For a Theory of Universals. Australasian Journal of Philosophy. 1983;61:343-377.

[10] Koslicki K. The structure of objects. Oxford University Press; 2008.

[11] Armstrong DM. A world of states of affairs. Cambridge University Press; 2008.

[12] Mormann T. On the mereological structure of complex states of affairs. Synthese. 2012;187(2):403-418.

[13] Kalhat J. Structural Universals and the Principle of Uniqueness of Composition. Grazer Philosophische Studien. 2008;76:57-77.

[14] McFarland A. Causal powers and isomeric chemical kinds. Synthese. 2018 Apr;195(4):1441-1457. 